\title{
Systematic dependences of the elliptic flow
}

\author{
Maya Shimomura for the PHENIX Collaboration ${ }^{* \dagger}$ \\ Iowa State Univ. \\ E-mail: mayapdrcf.rhic.on1.gov
}

\begin{abstract}
The transverse momentum $\left(p_{\mathrm{T}}\right)$ and centrality dependence of the azimuthal anisotropy $\left(v_{2}\right)$ are measured for charged hadron species at $\sqrt{s_{\mathrm{NN}}}=62.4$ and $200 \mathrm{GeV}$ in $\mathrm{Cu}+\mathrm{Cu}$ and $\mathrm{Au}+\mathrm{Au}$ collisions and at $\sqrt{s_{\mathrm{NN}}}=7.7$ and $39 \mathrm{GeV}$ in $\mathrm{Au}+\mathrm{Au}$ collisions by the PHENIX experiment at RHIC. The results are consistent with eccentricity scaling and with quark number $+\mathrm{KE}_{\mathrm{T}}$ scaling. Also, we found that $v_{2}$ divided by the participant eccentricity of the initial geometry proportionally increases with the number of participants to the $1 / 3$ power except at small $N_{\text {part }}$ in $\mathrm{Cu}+\mathrm{Cu}$ at 62.4 GeV. Taking these scaling (quark number, $\mathrm{KE}_{\mathrm{T}}$, eccentricity and $N_{\text {part }}^{1 / 3}$ ) into account, there is a universal scaling for $v_{2}$ with different energies, collision sizes and particle species. We also report that this scaling works at the LHC energy comparing PHENIX results to ALICE results. The results indicate that $v_{2}$ is determined by more than just the geometrical eccentricity and also depends on the size of collisions.
\end{abstract}

The Seventh Workshop on Particle Correlations and Femtoscopy

September 20th to 24th, 2011

The University of Tokyo, Japan

* Speaker.

${ }^{\dagger}$ The PHENIX Collaboration 


\section{Introduction}

Relativistic heavy ion collisions have been considered as a unique way to create the quarkgluon plasma (QGP), which is the phase of de-confined quarks and gluons. The Relativistic Heavy Ion Collider (RHIC) at Brookhaven National Laboratory was constructed to create and study the QGP. Azimuthal anisotropy of particles produced in relativistic heavy ion collisions is the one of the most powerful probes for investigating the characteristics of the QGP. Especially the strength of the elliptic anisotropy $\left(v_{2}\right)$, which is defined by the second harmonics of Fourier expansion for the azimuthal distribution of produced particles with respect to the reaction plane, is expected to be sensitive to the early stage of heavy ion collisions. At non-central collision, the overlap region is geometrically anisotropic, like an almond shape. When the produced matter has small mean free path, interacting each other enough to reach local thermalization, it creates pressure gradient. The geometrical anisotropy transfers to the anisotropy in the momentum phase space as flow because of this pressure gradient, and $v_{2}$ indicates the strength of this elliptic flow. Thus, the measured $v_{2}$ reflects the equation of state of the dense matter such as QGP, produced in the collisions. The important thing here is since the produced particles randomly emit before thermalization, the geometrical eccentricity decrease with time. Therefore, to let the geometrical eccentricity make elliptic flow, the thermalization should be occurred very early stage before the geometrical eccentricity is totally gone.

\section{Motivation}

One of the most remarkable findings at RHIC is that the strength of $v_{2}$ can be described well by hydro-dynamical models in the low transverse momentum region $(\sim 1 \mathrm{GeV} / c)$ [四]. In the intermediate transverse momentum region $(1 \sim 4 \mathrm{GeV} / c), v_{2}$ is consistent with $n_{\mathrm{q}}$ and $\mathrm{KE}_{\mathrm{T}}(=$ $\left.m_{\mathrm{T}}-m_{0}\right)$ scaling, and the result supports a quark-recombination model [ [ $]$ ]. The matter produced in the high energy heavy ion collision is expected to undergo several stages from the initial hard scattering to the final hadron emission. When the matter reaches thermalization and QGP is created, we expect hydro-dynamical behavior at quark level. Since experimentally we cannot see the QGP directly, we need a comprehensive understanding from thermalization through hadronization to freeze-out. The elliptic flow is expected to be created at QGP stage by pressure gradient, but it is important to note whenever the matter interacts with each other, there is a possibility to change $v_{2}$. [B]

For a more comprehensive understanding of $v_{2}$ production mechanism, we have carried out systematic measurements of $v_{2}$, by measuring $v_{2}$ for identified charged hadrons in Au+Au and $\mathrm{Cu}+\mathrm{Cu}$ collisions at $\sqrt{s_{\mathrm{NN}}}=200$ and $62.4 \mathrm{GeV}$ and also for inclusive charged hadrons in $\mathrm{Au}+\mathrm{Au}$ at $\sqrt{s_{\mathrm{NN}}}=7.7$ and $39 \mathrm{GeV}$. We have studied the dependence on collision energy, size and species of the produced particles comparing the $\sqrt{s_{\mathrm{NN}}}=2.76 \mathrm{TeV}$ data from LHC. [5]

\section{Results}

\subsection{Collision Energy Dependence}

In $\mathrm{Au}+\mathrm{Au}$ collisions, the values of $v_{2}$ as a function of $p_{\mathrm{T}}$ agree well at $\sqrt{s_{\mathrm{NN}}}=39,62.4$ and 

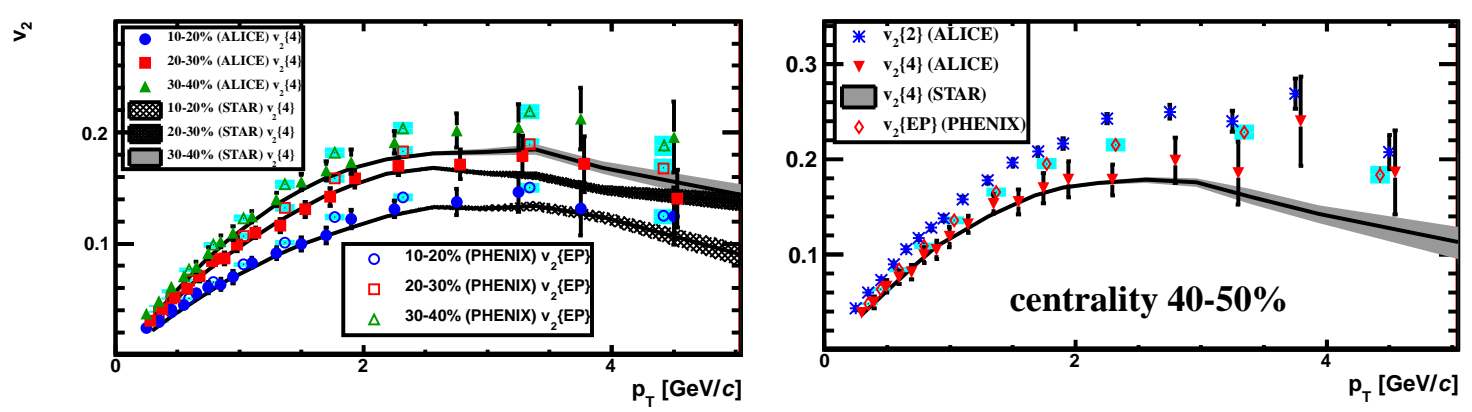

Figure 1: $v_{2}$ as a function of $p_{\mathrm{T}}$ at $\sqrt{s_{\mathrm{NN}}}=200 \mathrm{GeV}$ and $2.76 \mathrm{TeV}$. Left panel is for 10-20, 20-30 and 30-40 $\%$ and right panel is for $40-50 \%$. PHENIX results are measured by event plan method. ALICE and STAR results are measured by cumulant method. The detail of measurement are in [G] [G] [G].

$200 \mathrm{GeV}$ for measured centralities, 10-20, 20-30, 30-40 and 40-50\%. However, the $v_{2}$ at $7.7 \mathrm{GeV}$ is much lower than these. This results may indicate the energy between 7.7 and 39 is the region which switch from partonic flow to hadronic flow.

Additionally, we compared the results of $\sqrt{s_{\mathrm{NN}}}=2.76 \mathrm{TeV}$ data in $\mathrm{Pb}+\mathrm{Pb}$ at LHC-ALICE, and it was found that $v_{2}$ at $2.76 \mathrm{TeV}$ is very similar to the $v_{2}$ at $200 \mathrm{GeV}$ especially at low $p_{\mathrm{T}}$ as shown in Figure [. [[5] [ [G] [四] While the differential $v_{2}$ is consistent between LHC and RHIC, integrated $v_{2}$ is increasing at higher collision energy. This is because the spectra shape is flatter so that the mean $p_{\mathrm{T}}$ is higher at LHC. This flatter spectra has been considered to be caused by larger radial flow. However, if this is due to the radial flow, the differential $v_{2}$ should also be shifted to higher $p_{\mathrm{T}}$, so that the differential $v_{2}$ should be lower at low $p_{\mathrm{T}}$ at LHC and should not be matched to RHIC. Therefore, if we could subtract the effect from the radial flow, the $v_{2}$ values may not agree between LHC and RHIC.

\subsection{Eccentricity and $\mathbf{N}_{\text {part }}$ Scaling}

Next, we compared different system size of collisions. The left panel in Figure $\square$ shows the comparison of $v_{2}$ in $\mathrm{Au}+\mathrm{Au}, \mathrm{Cu}+\mathrm{Cu}$ and $\mathrm{Pb}+\mathrm{Pb}$ at $\sqrt{s_{\mathrm{NN}}}=200 \mathrm{GeV}, 62.4 \mathrm{GeV}$ and $2.76 \mathrm{TeV}$ as a function of $\mathrm{N}_{\text {part }}$. The values of $v_{2}$ agree well at $\sqrt{s_{\mathrm{NN}}}=200 \mathrm{GeV}, 62.4 \mathrm{GeV}$ and $2.76 \mathrm{TeV}$ in the errors, but have clear differences between the $\mathrm{Cu}+\mathrm{Cu}$ and $\mathrm{Au}+\mathrm{Au}(\mathrm{Pb}+\mathrm{Pb})$. This is natural because the different nucleus collisions such as $\mathrm{Au}+\mathrm{Au}, \mathrm{Cu}+\mathrm{Cu}$ and $\mathrm{Pb}+\mathrm{Pb}$, have different initial geometrical eccentricities at the same $N_{\text {part }}$. Normalizing $v_{2}$ by eccentricity, $\varepsilon$, (eccentricity scaling), all results follow one curve as shown in the middle panel in Figure $\square$, therefore, $v_{2}$ is scaled by the eccentricity at the same $\mathrm{N}_{\text {part }}$. Here, we use the participant eccentricity, which includes the effect of participant fluctuations [团]. One can see that the values of $v_{2} / \varepsilon$ are not a constant and it depends on $N_{\text {part }}$. Therefore, $v_{2}$ can be normalized by $\varepsilon$ at the same $N_{\text {part }}$, but $\varepsilon$ is not enough to determine $v_{2}$. We found that $v_{2} / \varepsilon$ is proportional to $N_{\text {part }}^{1 / 3}$ as shown in the right panel of Figure $\square$. Including results of $\sqrt{s_{\mathrm{NN}}}=2.76 \mathrm{TeV}, v_{2} /\left(\varepsilon \cdot N_{\text {part }}^{1 / 3}\right)$ is independent of the collision systems except for small $N_{\text {part }}$ in $\mathrm{Cu}+\mathrm{Cu}$ at $\sqrt{s_{\mathrm{NN}}}=62.4 \mathrm{GeV}$. This exception might indicate that this is a region where the matter has not reached sufficient thermalization, although the errors are too large to discuss the difference. Therefore, a scan of collision energies and sizes would be very important for further study of this exception. Figure $\square$ is for $p_{\mathrm{T}}=0.2-1.0 \mathrm{GeV} / c$. The results for $p_{\mathrm{T}}=1.0-2.0$ and $2.0-4.0 \mathrm{GeV} / c$ 

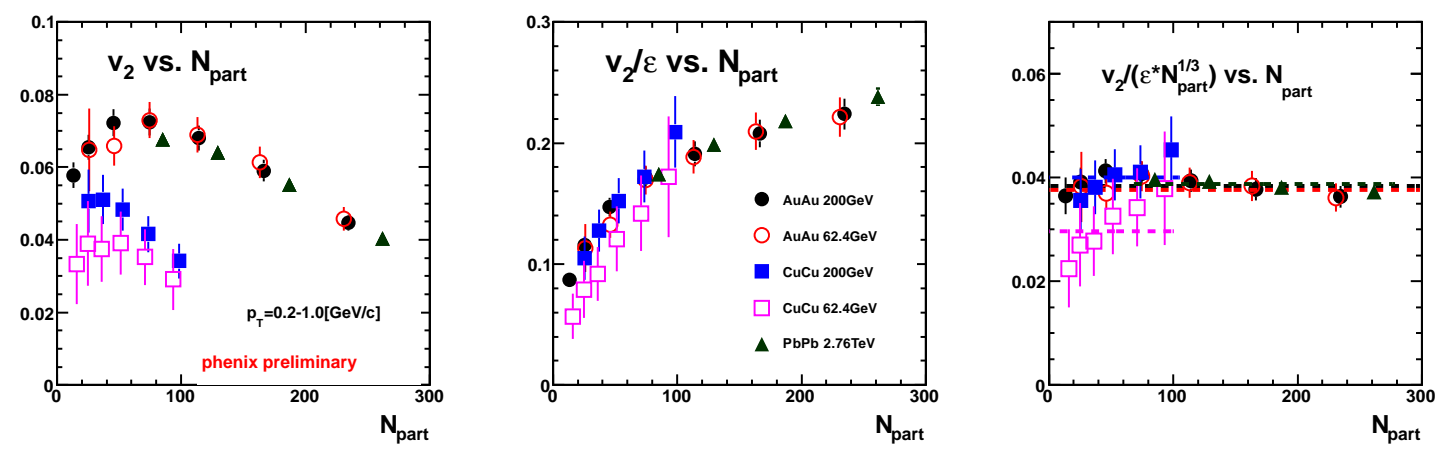

Figure 2: Comparison of integrated $v_{2}$ as a function of $N_{\text {part }}$ for five different collision systems. Left panel shows $v_{2}$ vs. $N_{\text {part }}$, middle panel is $v_{2} / \varepsilon$ vs. $N_{\text {part }}$ and right panel is $v_{2} /\left(\varepsilon \cdot N_{\text {part }}^{1 / 3}\right)$ vs. $N_{\text {part }}$ Closed symbols indicate the results of $\sqrt{s_{\mathrm{NN}}}=200 \mathrm{GeV}$ and $2.76 \mathrm{TeV}$, and open symbols are for $\sqrt{s_{\mathrm{NN}}}=62.4 \mathrm{GeV}$. Circles, squares and triangles indicate $\mathrm{Au}+\mathrm{Au}, \mathrm{Cu}+\mathrm{Cu}$ and $\mathrm{Pb}+\mathrm{Pb}$ collisions respectively. The statistical and systematic errors are included in the bars.

have the same tendency as well.

\subsection{Quark number+ $\mathbf{K E}_{\mathrm{T}}$ scaling and Universal $v_{2}$}

One of the most famous results on RHIC $v_{2}$ measurement is that the $v_{2}$ for quark number $\left(n_{\mathrm{q}}\right)+$ $\mathrm{KE}_{\mathrm{T}}$ scaling in $\mathrm{Au}+\mathrm{Au}$ at at $\sqrt{s_{\mathrm{NN}}}=200 \mathrm{GeV}$.[[]] The $n_{\mathrm{q}}$ scaling is consistent to the recombination model which assumes the quark level flow at QGP phase, and the $\mathrm{KE}_{\mathrm{T}}$ scaling has been considered to be able to subtract the difference of different particle $v_{2}$ at low $p_{\mathrm{T}}$ which is caused due to the radial flow effect. In $\mathrm{Au}+\mathrm{Au} 200 \mathrm{GeV}$ collisions at recent PHENIX experiment, the large statistics and new detector allowed us to see that the both $n_{\mathrm{q}}$ and $\mathrm{KE}_{\mathrm{T}}$ scaling works very well on various particle species including $\phi, \Lambda$ and deuteron, and even to see the break point of this scaling at $\mathrm{KE}_{\mathrm{T}}$ $=1 \mathrm{GeV}$ as shown in [Q] . Above this $p_{\mathrm{T}}$ region, one can expect other mechanism is dominant to create $v_{2}$.
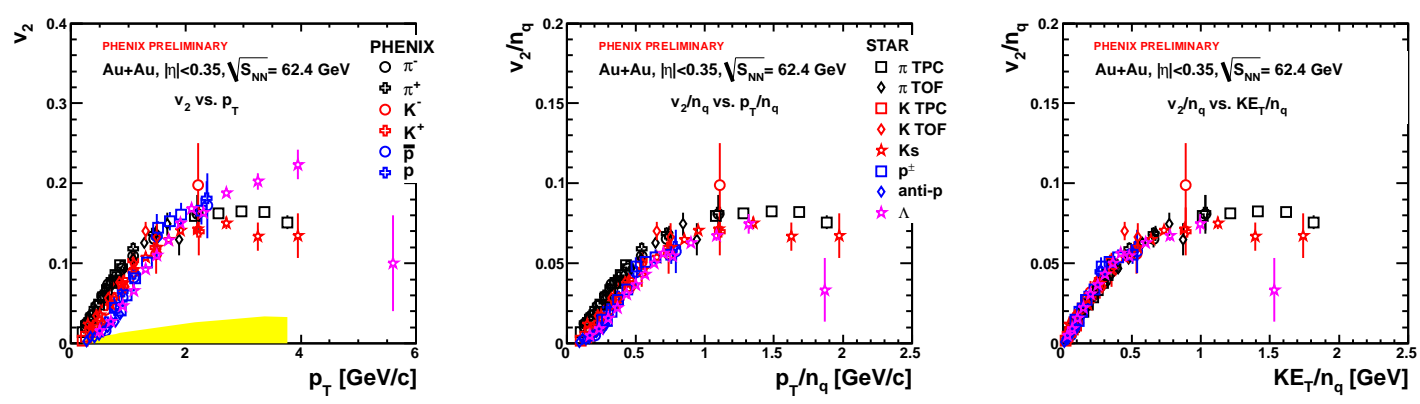

Figure 3: $v_{2}$ with the scaling. The left panel shows $v_{2}$ vs. $p_{\mathrm{T}}$, the middle shows $v_{2} / n_{q}$ vs. $p_{\mathrm{T}} / n_{q}$ and the left shows $v_{2} / n_{q}$ vs. $\mathrm{KE}_{\mathrm{T}} / n_{q}$ for various charged hadron species in $\mathrm{Au}+\mathrm{Au}$ at $\sqrt{s_{\mathrm{NN}}}=62 \mathrm{GeV}$ at $10-40 \%$ centrality bin.

For the systematic study, we also measured particle identified $v_{2}$ in $\mathrm{Au}+\mathrm{Au}$ at $\sqrt{s_{\mathrm{NN}}}=62.4$ $\mathrm{GeV}$ and in $\mathrm{Cu}+\mathrm{Cu}$ at $\sqrt{s_{\mathrm{NN}}}=200 \mathrm{GeV}$. The left panel of Figure [3 shows $v_{2}$ vs. $p_{\mathrm{T}}$, the middle shows $v_{2} / n_{q}$ vs. $p_{\mathrm{T}} / n_{q}$ and the left shows $v_{2} / n_{q}$ vs. $\mathrm{KE}_{\mathrm{T}} / n_{q}$ for various charged hadron species in $\mathrm{Au}+\mathrm{Au}$ at $\sqrt{s_{\mathrm{NN}}}=62 \mathrm{GeV}$ at $10-40 \%$ centrality bin. It is found that $v_{2}$ in $\mathrm{Au}+\mathrm{Au}$ at $62.4 \mathrm{GeV}$ 


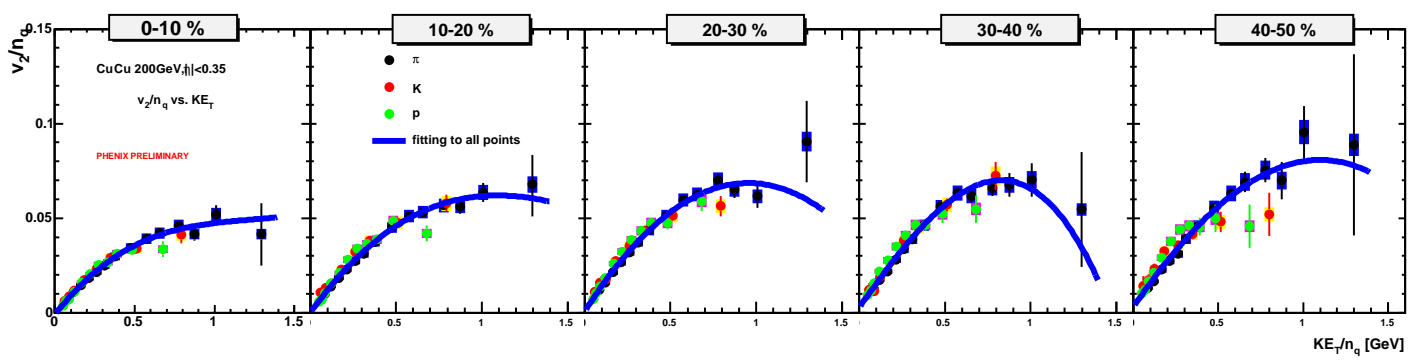

Figure 4: $v_{2} / n_{\mathrm{q}}$ vs. $\mathrm{KE}_{\mathrm{T}} / n_{\mathrm{q}}$ for charged $\pi, \mathrm{K}$ and $\mathrm{p}$ in $\mathrm{Cu}+\mathrm{Cu}$ at $\sqrt{s_{\mathrm{NN}}}=200 \mathrm{GeV}$ for $0-50 \%$ centrality bins as $10 \%$ step. Blue lines are the fitting to these scaled $v_{2}$ together by one curve at each centrality bin.

is also consistent with $n_{\mathrm{q}}+\mathrm{KE}_{\mathrm{T}}$ scaling. Moreover, as shown in Figure $\mathbb{\text { T the }} n_{\mathrm{q}}+\mathrm{KE}_{\mathrm{T}}$ scaling mostly works out in $\mathrm{Cu}+\mathrm{Cu}$ at $\sqrt{s_{\mathrm{NN}}}=200 \mathrm{GeV}$ for central collisions. However, you can see the small discrepancy from the $\mathrm{KE}_{\mathrm{T}}$ scaling at peripheral collisions at low $p_{\mathrm{T}}, 30-40$, and 40-50\%. For quantitative comparison, Figure $\square$ and $\$$ show the ratio of the $\pi / \mathrm{K} / \mathrm{p}$ scaled $v_{2}$ to the fitting function which fit these scaled v2 by one curve at each centrality bin. The fitting curves in $\mathrm{Cu}+\mathrm{Cu}$ at $\sqrt{s_{\mathrm{NN}}}$ $=200 \mathrm{GeV}$ are shown as the blue lines in Figure $\mathrm{H}$. To compare $\mathrm{Cu}+\mathrm{Cu}$ and $\mathrm{Au}+\mathrm{Au}$ Collisions, the values of $N_{\text {part }}$ at each centrality bin are written in each panel. It is clear that the discrepancy from the $\mathrm{KE}_{\mathrm{T}}$ scaling depends on $N_{\text {part }}$. Comparing between $\pi$ and proton, the results indicate the larger $N_{\text {part }}$ produces more shift for proton to higher $p_{\mathrm{T}}$ based on $\pi$. This $N_{\text {part }}$ dependence of $\mathrm{KE}_{\mathrm{T}}$ scaling behavior for the $v_{2}$ is explained by the radial flow effect with blast wave model in [Q].

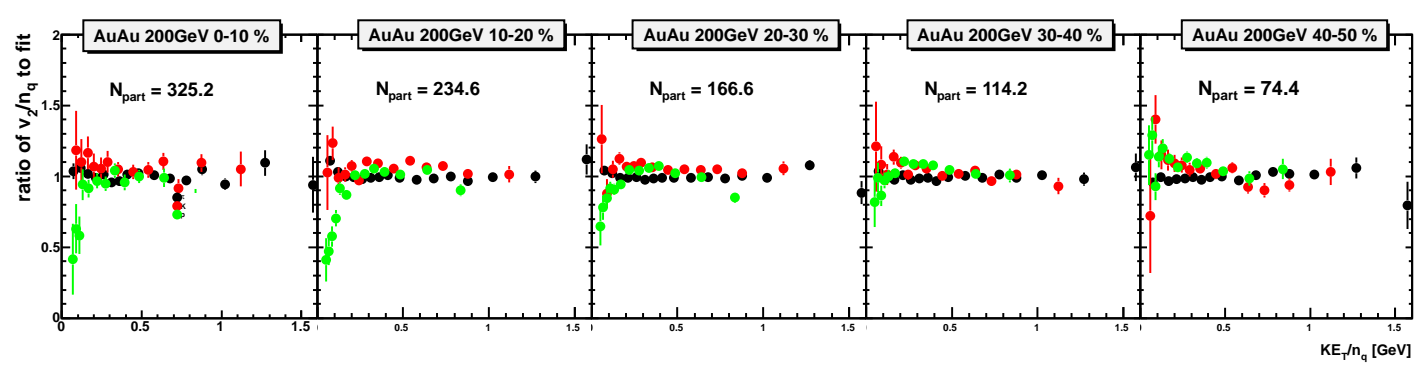

Figure 5: The ratio of the $\pi / \mathrm{K} / \mathrm{p}$ scaled $v_{2}$ to the fitting function which fit these scaled $\mathrm{v} 2$ by one curve at each centrality bin in $\mathrm{Au}+\mathrm{Au}$ at $\sqrt{s_{\mathrm{NN}}}=200 \mathrm{GeV}$.

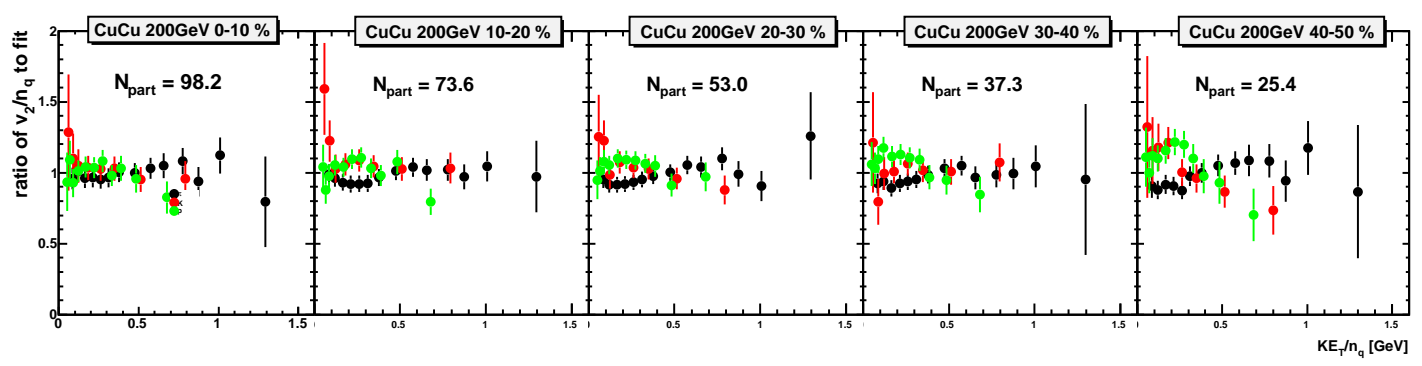

Figure 6: The ratio of the $\pi / \mathrm{K} / \mathrm{p}$ scaled $v_{2}$ to the fitting function which fit these scaled v2 by one curve at each centrality bin in $\mathrm{Cu}+\mathrm{Cu}$ at $\sqrt{s_{\mathrm{NN}}}=200 \mathrm{GeV}$. The fitting curves are shown as the blue lines in Figure 4. 

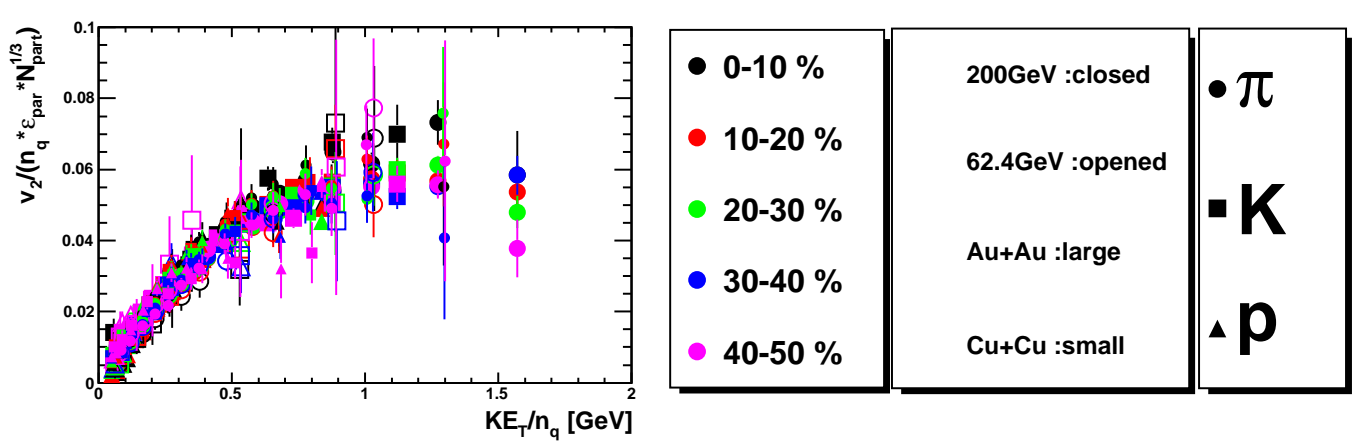

Figure 7: $v_{2} /\left(\varepsilon \cdot N_{\text {part }}^{1 / 3} \cdot n_{\mathrm{q}}\right)$ vs. $\mathrm{KE}_{\mathrm{T}} / n_{\mathrm{q}}$ for $\pi / \mathrm{K} / \mathrm{p}$ in $\mathrm{Au}+\mathrm{Au}$ at $\sqrt{s_{\mathrm{NN}}}=200 \mathrm{GeV}$, in $\mathrm{Au}+\mathrm{Au}$ at $\sqrt{s_{\mathrm{NN}}}=62.4$ $\mathrm{GeV}$ and in $\mathrm{Cu}+\mathrm{Cu}$ at $\sqrt{s_{\mathrm{NN}}}=200 \mathrm{GeV}$ at five centrality bins for $0-50 \%$ as $10 \%$ step for each system. There are 45 curves. Applying polynomial fitting, $\chi^{2} / \mathrm{NDF}$ is 2.1 .

In addition to the fact that $v_{2}\left(p_{\mathrm{T}}\right)$ is consistent at $\sqrt{s_{\mathrm{NN}}}=39-200 \mathrm{GeV}, v_{2}$ normalized by $n_{\mathrm{q}}+\mathrm{KE}_{\mathrm{T}}$, eccentricity, and $N_{\text {part }}^{1 / 3}$ scaling follows a universal curve as shown in the right panel of Figure $\square$. This figure includes the 45 curves for $\pi / \mathrm{K} / \mathrm{p}$ in $\mathrm{Au}+\mathrm{Au}$ at $\sqrt{s_{\mathrm{NN}}}=200 \mathrm{GeV}$, in $\mathrm{Au}+\mathrm{Au}$ at $\sqrt{s_{\mathrm{NN}}}=62.4 \mathrm{GeV}$ and in $\mathrm{Cu}+\mathrm{Cu}$ at $\sqrt{s_{\mathrm{NN}}}=200 \mathrm{GeV}$ for the five centrality bins from $0-50 \%$ in $10 \%$ steps. The $\chi^{2} / \mathrm{NDF}$ of the polynomial fitting is 2.1 . This is a universal scaling for $v_{2}$ with different energies, collision sizes and particle species, and it indicates that $v_{2}$ is determined not only by the geometrical eccentricity but also by the size of collision. This scaling assume that differential $v_{2}$ is consistent above $39 \mathrm{GeV}$ while " $v_{2} / \varepsilon$ vs. (1/S)(dN/dy)" scaling plotted in [ $[$ ] assumes that the higher collision energy produces higher $v_{2}$. Therefore, this $N_{\text {part }}^{1 / 3}$ scaling works better for the differential $v_{2}$ at PHENIX results. The size dependence of $v_{2}$ can be understood as thermal freeze-out nature of produced particles based on hydrodynamical behavior, which is different from that of chemical freeze-out. [Q]

\section{Acknowledgements}

I would like to express my great thanks to the organizers of this conference, WPCF 2011, for the opportunity to present these results.

\section{References}

[1] S. S. Adler et al., Phys. Rev. Lett. 91, 182301 (2003)

[2] A. Adare et al., Phys. Rev. Lett. 98, 162301 (2007)

[3] S. A. Voloshin, A. M. Poskanzer and R. Snellings (2008) nucl-ex/08092949

[4] B. Alver et al., Phys. Rev. Lett. 98, 242302 (2007)

[5] K. Aamodt et al., Phys.Rev.Lett 105, 252302 (2010)

[6] S. Afanasiev et al., Phys. Rev. C 80, 024909 (2009)

[7] B. I. Abelev et al., Phys. Rev. C 77, 054901 (2008)

[8] S. A. Voloshin for the STAR Collaboration, J. Phys. G 34, S883-886, (2007)

[9] M. Shimomura for the PHENIX Collaboration, J. Phys.: Conf. Ser. 270012041 (2011) 